\title{
The Lipgene Food-Exchange Model: a tool to enable investigation of four diets distinct in fatty acid composition
}

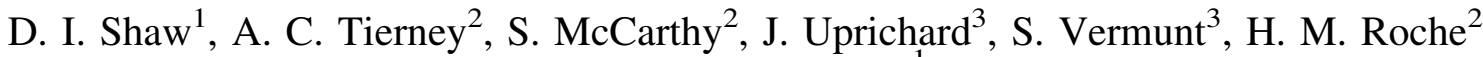 \\ and J. A. Lovegrove ${ }^{1}$ \\ ${ }^{1}$ School of Food Biosciences, University of Reading, RG6 6AP, ${ }^{2}$ Nutrigenomics Research Group, School of Medicine, \\ Trinity College Dublin Institute of Molecular Medicine, St James's Hospital, Dublin 8, Ireland and ${ }^{3}$ Unilever Health \\ Institute, Unilever Bestfoods, The Netherlands
}

The metabolic syndrome affects a substantial percentage of the European population (10-20\%). Evidence suggests that the presence of the metabolic syndrome increases the risk of type 2 diabetes mellitus (T2DM) and CVD by $2.8-8$ fold and $1.5-6$ fold, respectively ${ }^{(1)}$. In addition, global incidence of obesity is escalating at a phenomenal rate. It is unlikely (even with extreme public health intervention) that the obesity epidemic can be reversed in the short term. Thus, there is an urgent need for a treatment strategy for the metabolic syndrome and its associated risk of T2DM and CVD.

Human prospective evidence supports the hypothesis that dietary fat quality may influence insulin action, a central component of the metabolic syndrome ${ }^{(2)}$. However, controlled human intervention trials are required to confirm this hypothesis and causality. The aim of the current work was to develop a food-exchange model to enable the investigation of the effects of four experimental diets (A-D), distinct in fat quantity and quality and suitable for use in eight centres across Europe. Diets A and B were designed to provide $38 \%$ energy intake from fat with specific targets for SFA, MUFA and PUFA as 16\%, 12\%, 6\% and 8\%, 20\%, 6\%, respectively. Diets C and D were designed as low fat diets (28\% energy from fat) with target SFA, MUFA and PUFA set at $8 \%, 11 \%$ and $6 \%$. Diet D included a $1.24 \mathrm{~g}$ supplement of long chain $n$-3 PUFA/day. Diet C included a placebo capsule.

A food exchange model was developed based on the National Diet and Nutrition Survey of UK adults and a previously published strategy ${ }^{(3,4)}$. This was verified using Irish food consumption survey data. The amount of exchangeable fat in the average UK diet was calculated as the sum of fat provided by added fats (spreads and oils), milk, cheese, biscuits, cakes, buns and pastries. This was subtracted from total fat intake to determine unchangeable fat intake, i.e. that which could not easily be altered without severely affecting habitual dietary habits. This formed the backbone of the food exchange model. Subsequently, specifically designed sources of exchangeable fat were added to the basic model to create diets of differing overall composition. The study foods, provided by Unilever Bestfoods (The Netherlands), included spreads, cooking oils, mayonnaises, baking fats and biscuits. Also critical to the food-exchange model were the use of carbohydrate exchanges to ensure all four diets were isoenergtic.

Subjects completed 3-d weighed food diaries and FFQ to assess habitual dietary intake. Based on these, nutritionists advised subjects individually on recommended food choices for their allocated diet. Fortnightly $24 \mathrm{~h}$ recalls were taken and the frequency of study food consumption was monitored.
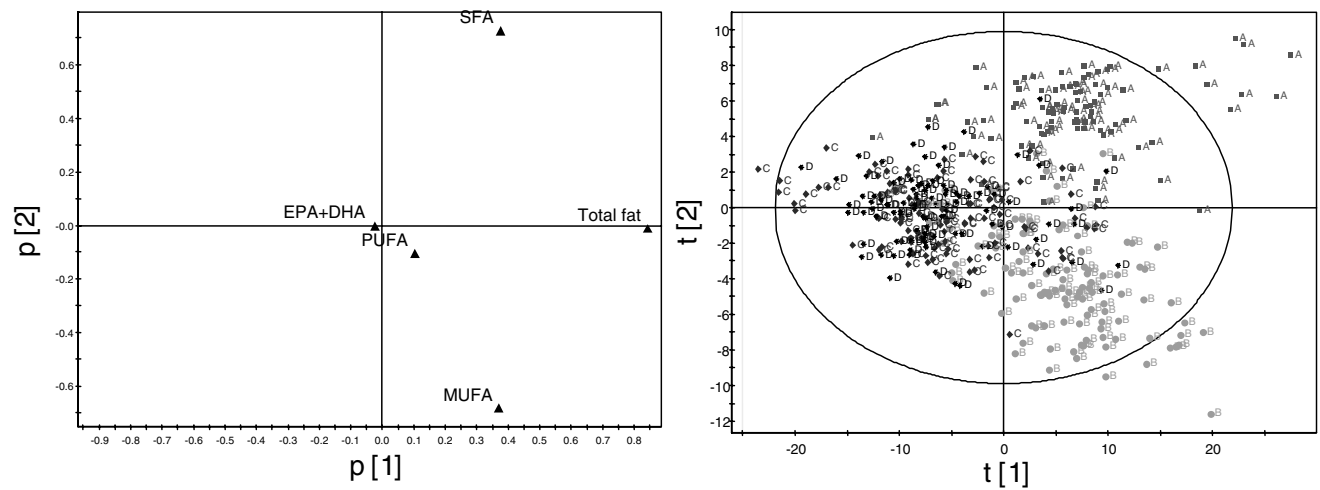

Figure 1: Four diets (A-D) distinct in fat composition at end of 12 week dietary intervention period. Loadings plot (left) shows dietary factors responsible for the positioning of data in the scores plot (right). Diet A is distinct in SFA, Diet B in MUFA and Diets C and D are low in total fat.

Initial data suggest that this strategy was successful in creating four diets, distinct in dietary fat content in the dietary intervention cohort (figure 1). This work was funded by the EC, Framework Programme 6 via the LIPGENE project (FOOD-CT-2003-505944).

1. Shaw D, Hall W \& Williams C. Metabolic Syndrome (2005) Proc Nutr Soc. 2005 64, 349-357.

2. Salmeron J, Hu F, Manson J, et al. (2001) Am J Clin Nutr. 2001 73, 1019-1026.

3. Henderson L, Gregory J, Irving K \& Swan G (2003) The National Diet and Nutrition Survey: adults aged 19-64 years.

4. Williams C, Francis-Knapper J, Webb D et al. (1999) BJN 81, 421-423. 